\title{
Pharmacological management of non-alcoholic fatty liver disease: Atorvastatin versus pentoxifylline
}

\author{
RAMONA CIOBOAT $\breve{1}^{1 *}$, ALICE GĂMAN ${ }^{2}$, DIANA TRAŞCĂ ${ }^{1}$, ANCA UNGUREANU $^{2 *}$, \\ ANCA OANA DOCEA ${ }^{3}$, PAUL TOMESCU ${ }^{4 *}$, FLORIN GHERGHINA $^{5 *}$, ANDREEA LETITIA ARSENE $^{6 *}$, \\ CORIN BADIU $^{7}$, ARISTIDES M. TSATSAKIS ${ }^{8}$, DEMETRIOS A. SPANDIDOS ${ }^{9}$, \\ NIKOLAOS DRAKOULIS ${ }^{10}$ and DANIELA CĂLINA ${ }^{11}$ \\ Departments of ${ }^{1}$ Internal Medicine, ${ }^{2}$ Bacteriology, Virology and Parasitology, ${ }^{3}$ Toxicology, \\ ${ }^{4}$ Urology and ${ }^{5}$ Physiotherapy, University of Medicine and Pharmacy of Craiova, Craiova 200349; \\ Departments of ${ }^{6}$ Microbiology and ${ }^{7}$ Endocrinology, Carol Davila University of Medicine and Pharmacy, \\ Bucharest 030167, Romania; ${ }^{8}$ Laboratory of Toxicology, and ${ }^{9}$ Laboratory of Clinical Virology, Medical School, \\ University of Crete, Heraklion 71003; ${ }^{10}$ Resesarch Group of Clinical Pharmacology and Pharmacogenomics, \\ Faculty of Pharmacy, School of Health Sciences, National and Kapodistrian University of Athens, Athens 15771, Greece; \\ ${ }^{11}$ Department of Clinical Pharmacy, University of Medicine and Pharmacy of Craiova, Craiova 200349, Romania
}

Received January 5, 2017; Accepted March 9, 2017

DOI: $10.3892 / \mathrm{etm} .2017 .4256$

\begin{abstract}
In this study, we aimed to evaluate the efficacy of pentoxifylline and atorvastatin in the treatment of non-alcoholic fatty liver disease (NAFLD). The study included 98 patients with histologically confirmed NAFLD divided into 2 groups as follows: group I (57 dyslipidemic patients, receiving atorvastatin $20 \mathrm{mg} /$ day and group II (41 non-dyslipidemic patients, treated with pentoxifylline, $800 \mathrm{mg} /$ day). The present study was conducted for a mean of $32.8 \pm 3.4$ weeks. For all patients, we determined the body mass index, a liver biopsy was performed, and we measured the serum levels of alanine aminotransferase (ALT), aspartate aminotransferase (AST), gamma-glutamyl transpeptidase (GGT), alkaline phosphatase (ALP), total cholesterol (TC) and triglycerides (TG) at the beginning and at the end of the study period. The NAFLD activity score (NAS) was used to evaluate the liver biopsies for steatosis, fibrosis and necroinflammation. The patients in group I exhibited a considerable reduction in ALT, AST, GGT,
\end{abstract}

Correspondence to: Dr Anca Oana Docea, Department of Toxicology, University of Medicine and Pharmacy of Craiova, 2 Petru Rareş Street, Craiova 200349, Romania

E-mail: ancadocea@gmail.com

Dr Corin Badiu, Department of Endocrinology, Carol Davila University of Medicine and Pharmacy, 37 Dionisie Lupu Street, Bucharest 030167, Romania

E-mail: badicrin@yahoo.co.uk

*Contributed equally

Key words: non-alcoholic fatty liver disease, atorvastatin, pentoxifylline
TC, AP and TG levels $(\mathrm{P}<0.0001)$. Histologically, there were no changes in fibrosis and necroinflammation, although the extent steatosis was reduced. The improvement in the ALT, AST and GGT values $(\mathrm{P}<0.05)$ in group II were similar to those in group I; however, no statistically significant decrease was noted in the levels of ALP, TC and TG in this group. Our results thus demonstrated that atorvastatin attenuated steatosis and improved liver function parameters in patients with NAFLD associated with dyslipidemia. Similar results were obtained in the non-dyslipidemic patients administered pentoxifylline.

\section{Introduction}

Non-alcoholic fatty liver disease (NAFLD) is a condition characterized by the accumulation of large fat droplets in hepatocytes (hepatic steatosis) that usually appear in those without a history of alcohol abuse or known liver disease (1).

NAFLD is a metabolic disorder affecting obese or overweight individuals in particular, and is considered the main cause of chronic liver disease with an increasing incidence worldwide (2). NAFLD is considered to be the hepatic component of metabolic syndrome, which includes features such as obesity, hyperinsulinemia, peripheral insulin resistance, diabetes, dyslipidemia, and hormonal disturbances secondary to interaction between this syndrome and reproductive axis (3). In women with ovarian dysfunction, such us polycystic ovary syndrome (PCOS) and acne, the possibility of NAFLD occurrence is much higher, and approximately $7 \%$ of obese patients with PCOS also develop NAFLD associated with insulin resistance $(4,5)$. Environmental pollutants, particularly pesticides or solvents represent tangible NAFLD risk factors (6-8). Studies have demonstrated that NAFLD is an important risk factor for the development of primary liver cancer, mostly due to NAFLD-associated cirrhosis (cryptogenic cirrhosis) $(9,10)$. A high prevalence of NAFLDS occurs in hepatitis $C$ virus infec- 
tions where double antiviral therapy with peginterferon and ribavirin can have a significant impact on the progression of the disease $(11,12)$. Understanding the pathogenesis, biochemical parameters, histological grading and staging, and the management of NAFLD, are vital in clinical practice today.

The prevalence of NAFLD in the general population is not yet completely understood. NAFLD is the most common liver disorder in Western industrialized countries, affecting $20-40 \%$ of the population. The major risk factors for NAFLD are abdominal obesity, type 2 diabetes mellitus, dyslipidemia and metabolic syndrome (13). Some studies also demonstrate an association between cardiovascular disease and development of NAFLD (14). NAFLD is also associated with metabolic syndrome, insulin resistance being considered as the key mechanism leading to hepatic steatosis (15). Therefore, it is important to actively search for NAFDL in other conditions associated with insulin resistance, such as PCOS, acromegaly and psoriasis, and to consider liver function when treating the primary disorder.

In the management of patients with NAFLD there is a need for a multidisciplinary system, as apart from the standard treatment for liver disease, and also a need for the specific treatment of associated metabolic disturbances, such as obesity, hyperlipidemia, insulin resistance and type 2 diabetes mellitus. The aim of this planned, prospective and uncontrolled study was to evaluate the efficacy of atorvastatin and pentoxifylline in treating NAFLD.

\section{Materials and methods}

The present study included 98 patients with histologically confirmed NAFLD, admitted between October 2012 and January 2016 at the Department of Internal Medicine at Filantropia University Hospital (Craiova, Romania). Upon admission, a comprehensive medical history and full physical examination was carried out, including the determination of body mass index (mean BMI, $31.45 \pm 5.54 \mathrm{~kg} / \mathrm{m}^{2}$ ). There were 2 groups of patients: group I (57 dyslipidemic patients and group II (41 non-dyslipidemic patients). No differences in terms of age and gender between the 2 groups were observed. The results of biochemical tests upon admission are shown in Table I.

This study was carried out in accordance with the Helsinki Declaration of 1975, and was approved by the Review Ethics Board of the University Medicine and Pharmacy of Craiova and of the Filantropia University Hospital. All patients involved in this study signed a full informed consent. Taking into consideration ethical concerns and the overall poor consent to hepatic biopsies, we decided not to use placebos or controls in the present study; this is primarily due to the fact that it would involve a large number of patients having to undergo two liver biopsies while receiving no active treatment.

The patients were divided into 2 therapeutic groups as follows: group I, 57 dyslipidemic patients receiving atorvastatin $20 \mathrm{mg} /$ day; and group II, 41 non-dyslipidemic patients treated with pentoxifylline $800 \mathrm{mg} /$ day (400 mg twice daily).

The average duration of drug administration was $32.8 \pm 3.4$ weeks. According to the study design, the patients were subjected to a medical examination upon admission (T0), two regular medical examinations (T1 and T2) at 10 and
Table I. Mean values of biochemical parameters and BMI in therapeutic groups at inclusion.

\begin{tabular}{|c|c|c|c|c|c|}
\hline \multirow[b]{2}{*}{ Parameter } & \multicolumn{2}{|c|}{$\begin{array}{c}\text { Group I } \\
n=57\end{array}$} & \multicolumn{2}{|c|}{$\begin{array}{c}\text { Group II } \\
n=41\end{array}$} & \multirow[b]{2}{*}{ P-value } \\
\hline & Mean & $\mathrm{SD}$ & Mean & SD & \\
\hline ALT UI/dl & 82.64 & 13.82 & 82.60 & 12.82 & 0.9884 \\
\hline AST UI/dl & 83.13 & 20.36 & 91.47 & 14.17 & 0.0262 \\
\hline GGT UI/dl & 52.99 & 15.27 & 55.27 & 17.55 & 0.4951 \\
\hline $\mathrm{TC} \mathrm{mg} / \mathrm{dl}$ & 298.76 & 26.76 & 141.32 & 34.56 & $<0.0001$ \\
\hline TG mg/dl & 260.09 & 62.36 & 95.41 & 27.12 & $<0.0001$ \\
\hline ALP UI/dl & 209.20 & 61.23 & 165.61 & 52.45 & 0.0004 \\
\hline $\mathrm{G}(\mathrm{mg} / \mathrm{dl})$ & 117.40 & 27.78 & 103.85 & 19.95 & 0.0090 \\
\hline $\mathrm{BMI}\left(\mathrm{kg} / \mathrm{m}^{2}\right)$ & 31.347 & 5.341 & 29.056 & 6.884 & 0.0667 \\
\hline
\end{tabular}

SD, standard deviation; BMI, body mass index; G, glucose; ALT, alanine aminotransferase; AST, aspartate aminotransferase; GGT, gamma-glutamyl transpeptidase; TC, total cholesterol; TG, triglycerides; ALP, alkaline phosphatase. The results of Wilcoxon test for comparisons between groups I and II are presented as P-values.

20 weeks after the initial medical examination and one medical examination at the end of treatment at week 30 (T3).

All patients (51 males/47 females) were Caucasians with a mean age of 54/52 years and no active viral hepatitis and no history of drug and/or alcohol abuse.

The study group was selected using inclusion and exclusion criteria as follows: patients were included in the study if they were able to provide written informed consent, had been histologically confirmed to suffer from NAFLD and had no history of drug and/or alcohol abuse. The exclusion criteria were represented by the history of chronic intake or abuse of alcohol or active viral hepatitis.

In monitoring alcohol intake, we used a questionnaire (which was slightly modified) based on the Behavioral Risk Factor Surveillance System 2006 Questionnaire (16) taken at each medical examination. The infrequent consumption of small amounts of alcohol amounts was permitted, in the condition that this did not exceed $>2$ drinks per week, with each drink being defined as one standard US alcoholic drink (approximately $14 \mathrm{~g}$ ethanol i.e., $12 \mathrm{oz}$ of beer, $5 \mathrm{oz}$ of wine, or $1.5 \mathrm{oz}$ of liquor; $1 \mathrm{US} \mathrm{oz}=$ approximately $30 \mathrm{ml}$ ).

No restriction or modifications in lifestyle or diet were enforced on any patient, apart from any current recommendations made by their endocrinologist or cardiologist. BMI, serum levels of alanine aminotransferase (ALT), aspartate aminotransferase (AST), gamma-glutamyl transpeptidase (GGT), alkaline phosphatase (ALP), total cholesterol (TC) and triglycerides (TG), and blood glucose levels were determined for all patients upon admission, at each visit and at the end of treatment. The patients were sampled after at least $8 \mathrm{~h}$ of overnight fasting by standard venipuncture.

The patients underwent liver biopsy at the beginning and end of the present study; we deemed 2 weeks before or after the first and last visit to be a respectable and acceptable time interval for biopsy. Hepatic biopsy was carried out using the Menghini 
Table II. Mean values for NAS scores at T0 and T3.

\begin{tabular}{lcccccc}
\hline & \multicolumn{2}{c}{ Atorvastatin } & & \multicolumn{2}{c}{ Pentoxifylline } & \\
\cline { 2 - 3 } & Mean & SD & & Mean & SD & P-value \\
\hline Score 1 & 5.26 & 1.82 & & 5.06 & 1.65 & 0.5639 \\
Score 2 & 2.93 & 1.62 & & 3.98 & 1.48 & 0.0093 \\
Mean diff. & & & & & \\
Score 1/ Score 2 & \multicolumn{2}{c}{2.33} & & 1.08 & \\
P-value & $<0.001$ & & $<0.05$ & \\
\hline
\end{tabular}

NAS, NAFLD activity score; SD, standard deviation. The results of the Wilcoxon test for comparisons between the NAS scores between groups I and II at times T0 and T3 and for each group between times T0 and T3 are presented as P-values.

technique, using Braun Melsungen Sonocan $20 \mathrm{G}$ needles (B. Braun Medical S.R.L., Timis, Romania), 0.8x160 mm and $21 \mathrm{G}, 0.8 \times 160 \mathrm{~mm}$. Biopsy fragments with a minimum length of $12 \mathrm{~mm}$ were considered adequate and delivered for pathological analysis.

Histological staining techniques were carried out to determine inflammation, steatosis and hepatic fibrosis using the NAFLD activity score (NAS or the Brunt score) for each case, upon admission into the study and at the end of the treatment, as previously described (17).

In this study, we used the NASH recognized lesion evaluation system developed by Kleiner et al (17). Using these criteria, the NASH Clinical Research Network Pathology Committee designed and validated a histological system of scoring which addresses the entire spectrum of NAFLD lesions and proposed a NAS to use in clinical trials (17). The scoring system is made up of 14 histological features, of which 4 were evaluated semi-quantitatively: lobular inflammation (0-2), steatosis (0-3), fibrosis (0-4) and hepatocellular ballooning (0-2). NAS is the sum of lobular inflammation, steatosis and hepatocellular ballooning scores, a NAS score $>5$ correlates with a NASH diagnosis and scores of $\leq 3$ are considered as 'not NASH.' The NAS admission scores at T0 (score 1) and at the termination of the study at T3 (score 2) were database-stored and compared between the therapeutic groups.

The results were calculated as the means \pm standard deviation. For data processing, the program Microsoft Excel was used (Microsoft Corp., Redmond, WA, USA), along with the XLSTAT suite for MS Excel (Addinsoft SARL, Paris, France).

For statistical comparisons between the 2 groups at admission (T0) and at the end of the treatment period (T3), variance analysis (one way ANOVA) was used, and for comparisons within the groups themselves, variant analysis and the Kruskall-Wallis and Wilcoxon tests were used. A value of $\mathrm{P}<0.05$ was considered to indicate a statistically significant difference.

\section{Results}

During the analysis of our results, we noted that the TG and TC levels were higher in group I compared with group II at T0, while the ALT, AST, GGT and ALP values were comparable between the groups (Table I). BMI and blood glucose levels were also higher in the dyslipidemic patients than in the patients with normal lipid levels (Table I).

The mean NAS score upon admission (NAS score T0) was $5.26 \pm 1.82$ in group I and $5.06 \pm 1.48$ in group II, with no significant differences between the 2 therapeutic groups $(\mathrm{P}=0.5639, \mathrm{P}>0.05)$ (Table II); likewise, there were no significant differences observed between the 2 groups as regards steatosis, necroinflammation, ballooning and fibrosis upon admission (data not shown).

The importance of evaluating patients at $\mathrm{T} 1$ and $\mathrm{T} 2$, respectively, consisted of the information obtained on the direct and comparative effects of administering atorvastatin or pentoxifylline after 10 and 20 weeks following the different treatments (Table III). During treatment, an improvement in the liver biochemical parameters was noted.

Table III. Mean values of biochemical parameters and BMI in the therapeutic groups at the different time points in the experiment.

$$
\text { Group I }
$$

\begin{tabular}{lcccccccccc} 
Param & \multirow{2}{*}{ T0 } & T1 & T2 & T3 & P-value & T0 & T1 & T2 & T3 & P-value \\
\hline ALT & $82.64 \pm 13.82$ & $75.12 \pm 11.89$ & $69.53 \pm 10.20$ & $43.63 \pm 10.32<0.0001$ & $82.6 \pm 12.82$ & $80.02 \pm 12.19$ & $77.08 \pm 9.59$ & $54.9 \pm 6.83$ & $<0.0001$ \\
AST & $83.13 \pm 20.36$ & $75.59 \pm 16.57$ & $70.61 \pm 13.31$ & $43.28 \pm 12.35<0.0001$ & $91.47 \pm 14.17$ & $88.87 \pm 11.62$ & $86.91 \pm 9.11$ & $58.59 \pm 8.45$ & $<0.0001$ \\
GGT & $52.99 \pm 15.27$ & $49.82 \pm 12.26$ & $48.84 \pm 9.04$ & $37.02 \pm 10.45$ & $<0.0001$ & $55.27 \pm 17.55$ & $53.04 \pm 13.46$ & $51.23 \pm 11.34$ & $42.95 \pm 8.95$ & $<0.0001$ \\
TC & $298.76 \pm 26.76$ & $275.60 \pm 24.24$ & $269.72 \pm 20.77$ & $243.7 \pm 16.07$ & $<0.0001$ & $141.32 \pm 34.56$ & $141.85 \pm 29.41$ & $136.57 \pm 30.09$ & $137.68 \pm 31.79$ & 0.837 \\
TG & $260.09 \pm 62.36$ & $257.31 \pm 55.08$ & $249.96 \pm 46.13$ & $239.04 \pm 40.41$ & 0.002 & $95.41 \pm 27.12$ & $95.81 \pm 26.27$ & $91.09 \pm 22.19$ & $88.44 \pm 18.35$ & 0.435 \\
ALP & $209.2 \pm 61.23$ & $218.32 \pm 60.45$ & $178.93 \pm 54.89$ & $142.5 \pm 40.2$ & $<0.0001$ & $165.61 \pm 52.45$ & $202.06 \pm 68.35$ & $208.31 \pm 49.95$ & $151.07 \pm 40.98<0.0001$ \\
G & $117.4 \pm 27.78$ & $115.91 \pm 26.18$ & $111.35 \pm 25.24$ & $112.68 \pm 19.8$ & 0.542 & $103.85 \pm 19.95$ & $101.85 \pm 17.48$ & $103.88 \pm 15.42$ & $119.39 \pm 21.12<0.0001$ \\
BMI & $31.347 \pm 5.341$ & $31.47 \pm 5.65$ & $31.23 \pm 6.74$ & $30.337 \pm 4.341$ & 0.726 & $29.056 \pm 6.884$ & $28.75 \pm 6.324$ & $28.36 \pm 6.783$ & $28.059 \pm 5.867$ & 0.905
\end{tabular}

BMI, body mass index; G, glucose; ALT, alanine aminotransferase; AST, aspartate aminotransferase; GGT, gamma-glutamyl transpeptidase; TC, total cholesterol; TG, triglycerides; ALP, alkaline phosphatase. The results of ANOVA test for comparison between times T0, T1, T2 and T3, for each of the groups, are presented as P-values. Param, parameter. T0, upon admission; T1, at 10 weeks; T2, at 20 weeks; T3, at week 30. 
A

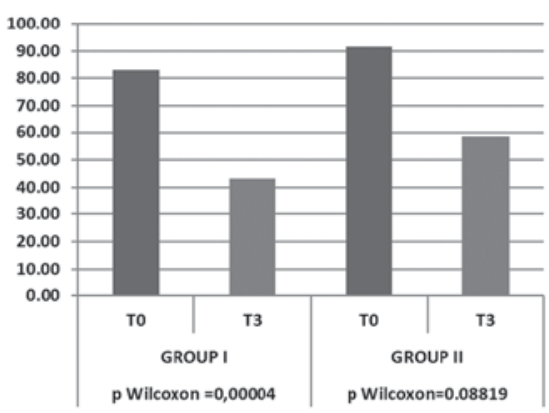

AST values TO-T3

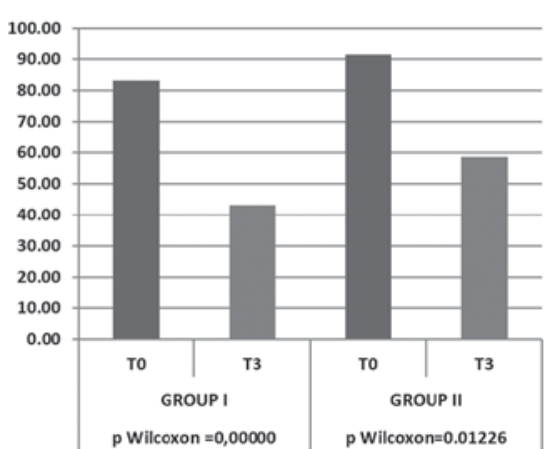

C

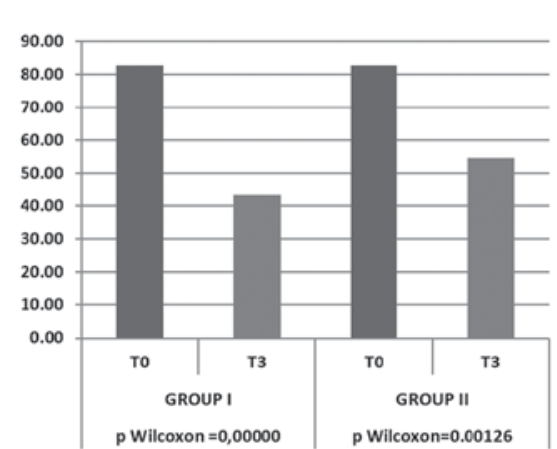

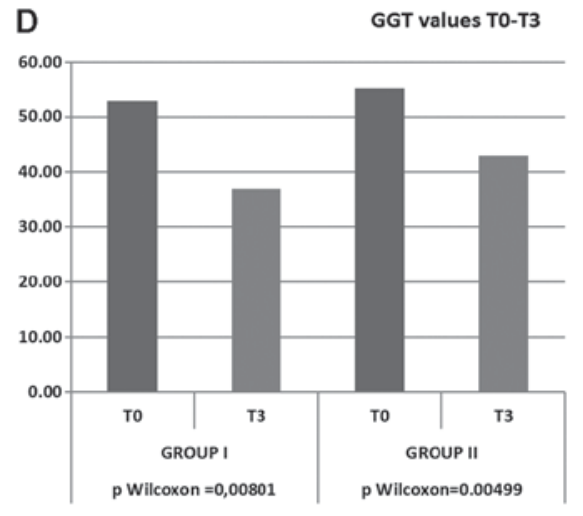

$\mathbf{F}$

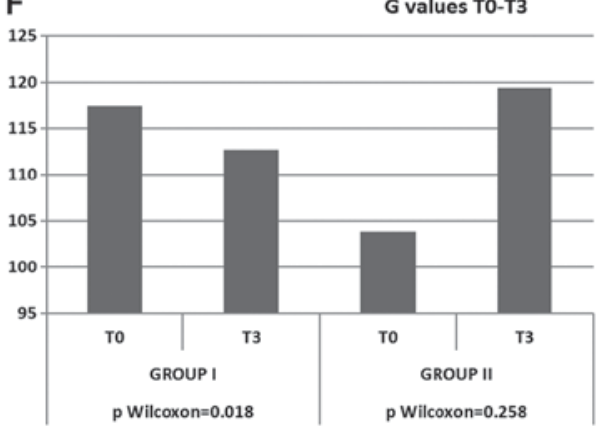

E

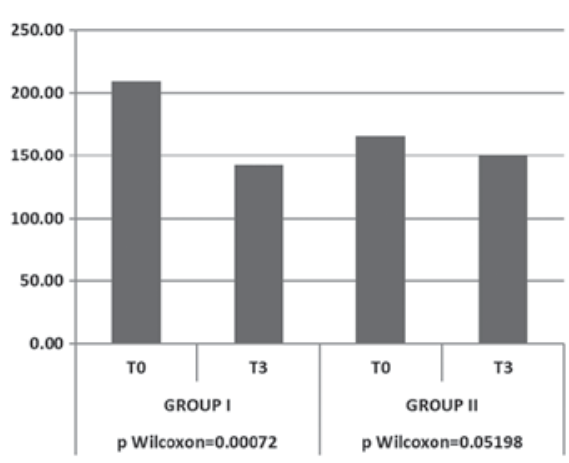

G

TG values T0-T3

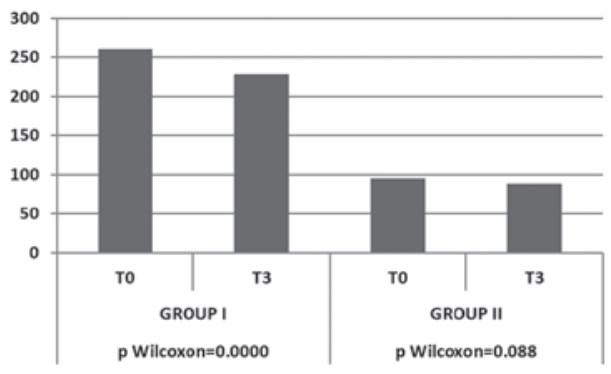

Figure 1. Variation of biochemical parameters T0-T3. (A) Total cholesterol (TC); (B) aspartate aminotransferase (AST); (C) alanine aminotransferase (ALT); (D) gamma-glutamyl transpeptidase (GGT); (E) alkaline phosphatase (ALP); F glucose (G) and (G) triglycerides (TG).

The results of biochemical parameters after 30 weeks from the beginning of the present study revealed a significant decrease $(\mathrm{P}=0.018)$ in the glucose levels in patients from study group I and a non-significant decrease in patients from study group II. A significant a decrease was observed in group I in the levels of ALT $(\mathrm{P}<0.0001)$, AST $(\mathrm{P}<0.0001)$, GGT $(\mathrm{P}<0.0001)$, TC $(\mathrm{P}<0.0001)$ and ALP $(\mathrm{P}<0.0001)$. In group II, the decrease in the ALT, AST and GGT levels was similar to that of group I $(\mathrm{P}<0.05)$; however, the levels of TC and TG in group II were only slightly and non-significantly decreased (Tables III and IV, and Fig. 1)..

No significant alteration was noticed regarding the levels of glucose and BMI within the groups. After the first 10 weeks of treatment, a significant decrease in the ALT levels in both groups was observed (Table III). In both groups, a significant decrease was observed in the ALT and AST levels; the ALT and
AST levels markedly decreased after 20 weeks of treatment. For GGT values, a similar descending trend was observed during treatment with the difference that lower rates were noted between T2 and T3. Histopathological evaluation for group I presented a mean NAS score at termination of $3.13 \pm 1.62$, which is highly significantly lower than score $1(\mathrm{P}=0.0007)$. Group II exhibited a mean NAS score at termination of $3.98 \pm 1.48$, which is borderline significant compared to the initial NAS score $(\mathrm{P}=0.04073)$. The lowest NAS score at termination was achieved by the patients in group I treated with atorvastatin (Table III).

NAS components improvement (one or more) was noticed only in group I; however, no attenuation of fibrosis was observed in either group. The steatosis score was significantly decreased in patients from group I, but only group II experienced an improvement of necroinflammation that was statistically 
Table IV. Mean values for biochemical parameters and BMI at study termination.

\begin{tabular}{lcccccc}
\hline & \multicolumn{2}{c}{ Atorvastatin } & & \multicolumn{2}{c}{ Pentoxifylline } & \\
\cline { 2 - 3 } Parameter & Mean & SD & & Mean & SD & P-value \\
\hline ALT & 43.63 & 10.32 & & 54.90 & 6.83 & $<0.0001$ \\
AST & 43.28 & 12.35 & & 58.59 & 8.45 & $<0.0001$ \\
GGT & 37.02 & 10.45 & & 42.95 & 8.95 & 0.0041 \\
TC & 243.70 & 16.07 & 137.68 & 31.79 & $<0.0001$ \\
TG & 239.04 & 40.41 & 88.44 & 18.35 & $<0.0001$ \\
ALP & 142.50 & 40.20 & 151.07 & 40.98 & 0.3044 \\
G & 112.68 & 19.80 & 119.39 & 21.12 & 0.1108 \\
BMI & 30.337 & 4.341 & 28.059 & 5.867 & 0.905 \\
\hline
\end{tabular}

SD, standard deviation; BMI, body mass index; G, glucose; ALT, alanine aminotransferase; AST, aspartate aminotransferase; GGT, gamma-glutamyl transpeptidase; TC, total cholesterol; TG, triglycerides; ALP, alkaline phosphatase. The results of Wilcoxon test for comparisons between groups I and II are presented as P-values.
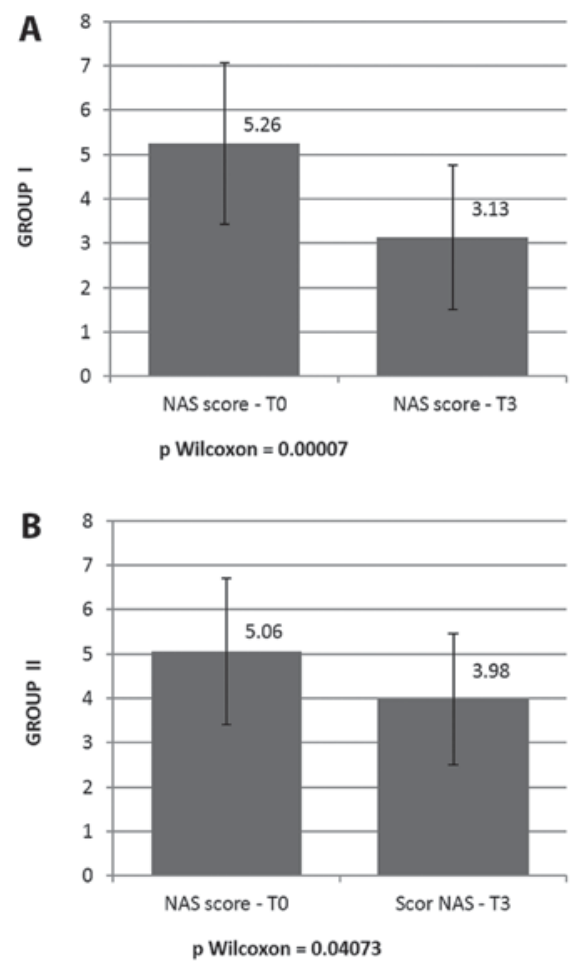

Figure 2. Mean values for NAS scores at T0 and T3 for (A) group I and (B) group II. NAS, NAFLD activity score. HS, highly significant; S, significant.

significant. A comparison between the NAS scores at admission vs. termination in both groups is shown in Fig. 2 .

\section{Discussion}

The pharmacological treatment of NAFLD includes vitamin E, insulin sensitisers and other metabolic agents aiming an antioxidant or hypolipemic activity, such as atorvastatin, while pentoxifylline inhibits the production of tumor necrosis factor- $\alpha$ (TNF- $\alpha$ ), which stimulates NASH development.

The results of the present study are in agreement with those of other trials. In short, both drugs led to a significant reduction in ALT and GGT levels. No significant weight loss was observed in the current study $(\mathrm{P}>0.05)$. Atorvastatin also led to a significant reduction in the levels of ALP $(\mathrm{P}<0.0001)$, TC $(\mathrm{P}<0.0001)$ and TG $(\mathrm{P}=0.002)$.

The lack of a control group imposed limits on the ability of determining the real pharmacotherapy impact, but when considering that these parameters failed to improve treatment previously, it is possible that the positive effects observed in the present study were caused by pentoxifylline and atorvastatin.

On the other hand, the histological evaluation showed significant improvement in 2 of the 4 NAS components of both groups. Neither group exhibited any changes in fibrosis. The dyslipidemic patients exhibited a highly statistically significant difference between the initial and final NAS scores, resulting in a P-value of 0.00007 . The difference in the initial and final NAS scores of patients from the non-dyslipidemic group administered pentoxifylline was of marginal significance $(\mathrm{P}=0.04073)$.

Atorvastatin is a HMG-CoA reductase inhibitor which catalyzes the HMG-CoA conversion into mevalonate, an early and rate-limiting step in the cholesterol biosynthesis, leading to low cholesterol production by the liver, high LDL cholesterol plasmatic clearance and up-regulation of hepatocyte LDL-receptors (18).

Kiyici et al observed that the use of atorvastatin for 6 months on a number of 27 dyslipidemic patients, for whom NASH diagnosis was histopathologicaly verified, was both effective and safe (19), while Balistreri observed that atorvastatin normalized serum ALT levels, TC and TG levels in patients with NASH (20).

In 2011, as part of the Saint Francis Heart Study, 80 patients with NAFLD confirmed by computer tomography were administered $20 \mathrm{mg} /$ day atorvastatin combined with $1 \mathrm{~g}$ vitamin C, and 1,000 IU vitamin E. After 4 years of therapy, the reduction of hepatic steatosis was $71 \%(\mathrm{OR}=0.29, \mathrm{P}<0.001)$. However, the fact that the patients received a combination of vitamins $\mathrm{C}$ and $\mathrm{E}$ along with atorvastatin, and that the NAFLD diagnosis was based on imaging and not on histology, limited the power of the conclusions (21).

Gómez-Domínguez et al examined the way in which atorvastatin in doses of $10-80 \mathrm{mg} /$ day affects lipid metabolism and transaminases levels in 22 patients for whom NAFLD diagnosis was established through ultrasonography. Following 6 months of treatment, $36.3 \%$ of the patients presented normal levels of transaminases and TC and after 12 months of treatment, a statistically noticeable decrease in transaminases levels and TC (from $268.5 \pm 44.2$ to $186.8 \pm 14.4 \mathrm{mg} / \mathrm{dl}$ ) were present, confirming the effect of atorvastatin in NAFLD and dyslipidemic patients without notable side-effects for daily doses of 10-80 mg (22).

Pentoxifylline is an inhibitor of TNF- $\alpha$. It is evident that TNF- $\alpha$ is associated with hepatic inflammatory cell recruitment, which represents a key step in the initiation and perpetuation of NASH liver injury (23). TNF- $\alpha$ also interferes with insulin receptor signaling by impairing and reducing insulin sensitivity.

Several pilot studies have demonstrated biochemical improvement, and in some cases histological improvement 
following the administration of pentoxifylline to patients with NASH (24-26).

Satapathy et al observed in 18 histopathologicaly verified NAFLD patients with elevated transaminases levels, that after 6 months of pentoxifylline administration in $800 \mathrm{mg} /$ day doses, the serum transaminases levels were significantly reduced (AST: $66 \pm 29$ to $33 \pm 11 \mathrm{IU} / 1, \mathrm{P}<0.0001$ and ALT: $109 \pm 44$ vs. $47 \pm 20$ IU/1, $\mathrm{P}<0.0001)$. Moreover, ALT was normalized for $23 \%$ of the patients within the first month $(\mathrm{P}=0.125)$, $35 \%$ after the second month and $60 \%$ of the patients after 6 months of treatment. The levels of cholesterol and TG were not significantly altered (24).

The same authors, reported in 2007 of nine patients that administered $800 \mathrm{mg}$ pentoxifylline daily doses and after an average of 12 months treatment, a decrease in transaminase values was achieved (ALT: $111 \pm 53$ to $45 \pm 19 \mathrm{IU} / 1, \mathrm{P}=0.003$ and AST: $61 \pm 27$ to $33 \pm 12 \mathrm{IU} / 1, \mathrm{P}=0.005$ ) accompanied by steatosis and lobular inflammation reduction in $55 \%$ of the cases, as well as decreased histological fibrosis in 4 out of 9 patients with baseline fibrosis (25).

In another study, on 55 patients with NASH confirmed by biopsy who received $400 \mathrm{mg}$ pentoxifylline 3 times per day or the placebo for 1 year, patients treated with pentoxifylline were more likely than those treated with the placebo to present a decrease in histological NAFLD. The NAS score decreased by 2 points in $38.5 \%$ of the patients treated with pentoxifylline compared to only $13.8 \%$ of the patients receiving the placebo. In this study, the administration of pentoxifylline improved the liver fibrosis scores, lobular inflammation and steatosis. In 3 patients receiving pentoxifylline, the dose of medication was decreased from 3 times daily to twice daily due to nausea, which resulted in adequate symptom control (26).

In another study by Van Wagner et al, in 30 patients treatesd with pentoxifylline, in doses of 1,200 $\mathrm{mg}$ /day, or placebo, for a period of 12 months. At the completion of the study, decreases in the transaminase levels (ALT: $92 \pm 12$ to $67 \pm 13$ IU/l and AST: $67 \pm 6$ to $47 \pm 6$ IU/1, $P<0.05)$, as well as in the degree of steatosis and lobular inflammation $(\mathrm{P}<0.05)$ were observed in the group administed pentoxifylline (27).

In other studies, pentoxifylline at doses of $400 \mathrm{mg} /$ day twice daily, administered to 20 patients for 12 months, was associated with the normalization of serum levels of ALT and AST ( $84 \pm 64$ vs. $138 \pm 76, P=0.002$ and $58 \pm 37$ vs. $102 \pm 62$, $\mathrm{P}=0.003$, respectively). A total of 9 patients withdrew from the study, due to nausea (28) and in another pilot study, similar biochemical improvements under pentoxifylline treatment were demonstated (29).

Neuner et al (30) studied the mechanisms of pentoxifylline action in NASH. All patients studied presented elevated levels of TNF- $\alpha$. Hepatic damage is associated with TNF- $\alpha$ production that triggers the production of various cytokines (31), recruiting inflammatory cells, that affect hepatocytes and induce fibrogenesis $(32,33)$. The main mechanism through which pentoxifylline improves hepatic histology (decreasing steatosis and necroinflammation) is the reduction of lipopolysaccharide stimulated TNF- $\alpha$ production.

In conclusion, statins are well known for their lipid lowering properties, but they may have the potential to diminish some of the histological features of NAFLD. Dyslipidemia is common among patients with NAFLD and atorvastatin proved to be efficient in the treatment of both disorders, by improving biochemical parameters and steatosis. Pentoxifylline was well-tolerated and showed similar efficacy in patients with non-dyslipidemia by decreasing the degree of steatosis/lobular inflammation and improving liver function.

\section{References}

1. Guturu P and Duchini A: Etiopathogenesis of nonalcoholic steatohepatitis: Role of obesity, insulin resistance and mechanisms of hepatotoxicity. Int J Hepatol 2012: 212865, 2012.

2. Bala C, Crăciun AE and Hâncu N: Updating the concept of metabolically healthy obesity. Acta Endo 12: 197-205, 2016.

3. Badiu C: Endocrine management in Prader-Willi Syndrome. Acta Endo 8: 99-106, 2012.

4. Ianoşi S, Ianoşi G, Neagoe D, Ionescu O, Zlatian O, Docea AO, Badiu C, Sifaki M, Tsoukalas D, Tsatsakis AM, et al: Age-dependent endocrine disorders involved in the pathogenesis of refractory acne in women. Mol Med Rep 14: 5501-5506, 2016.

5. Rehm JL, Connor EL and Reeder SB: Non-alcoholic fatty liver disease in an adolescent with polycystic ovary syndrome. $\mathbf{J}$ Pediatr Adolesc Gynecol 24: e61-e66, 2011.

6. Arciello M, Gori M, Maggio R, Barbaro B, Tarocchi M, Galli A and Balsano C: Environmental pollution: A tangible risk for NAFLD pathogenesis. Int J Mol Sci 14: 22052-22066, 2013.

7. Hernández AF, Gil F, Lacasaña M, Rodríguez-Barranco M, Tsatsakis AM, Requena M, Parrón T and Alarcón R: Pesticide exposure and genetic variation in xenobiotic-metabolizing enzymes interact to induce biochemical liver damage. Food Chem Toxicol 61: 144-151, 2013.

8. Tsitsimpikou C, Tzatzarakis M, Fragkiadaki P, Kovatsi L, Stivaktakis P, Kalogeraki A, Kouretas D and Tsatsakis AM: Histopathological lesions, oxidative stress and genotoxic effects in liver and kidneys following long term exposure of rabbits to diazinon and propoxur. Toxicology 307: 109-114, 2013.

9. Duan XY, Zhang L, Fan JG and Qiao L: NAFLD leads to liver cancer: do we have sufficient evidence? Cancer Lett 345: 230-234, 2014.

10. Baffy G, Brunt EM and Caldwell SH: Hepatocellular carcinoma in non-alcoholic fatty liver disease: an emerging menace. J Hepatol 56: 1384-1391, 2012.

11. Marconi A, Candido S, Talamini R, Libra M, Nicoletti F, Spandidos DA, Stivala F and Proietti L: Prevalence of hepatitis C virus infection among health-care workers: A 10 -year survey. Mol Med Rep 3: 561-564, 2010.

12. Docea AO, Gofiță E, Călina D, Zaharie SI, Vâlcea DI and Mitruț P: Autoimmune disorders due to double antiviral therapy with Peginterferon and Ribavirin in patients with hepatitis C virus infection. Farmacia 64: 605-611, 2016.

13. Chitturi S, Farrell GC, Hashimoto E, Saibara T, Lau GK and Sollano JD: Asia-Pacific Working Party on NAFLD: Non-alcoholic fatty liver disease in the Asia-Pacific region: Definitions and overview of proposed guidelines. J Gastroenterol Hepatol 22: 778-787, 2007.

14. Lizardi-Cervera J and Aguilar-Zapata D: Nonalcoholic fatty liver disease and its association with cardiovascular disease. Ann Hepatol 8 (Suppl 1): S40-S43, 2009.

15. Almeda-Valdés P, Cuevas-Ramos D and Aguilar-Salinas CA: Metabolic syndrome and non-alcoholic fatty liver disease. Ann Hepatol 8: S18-S24, 2009.

16. Centers for Disease Control and Prevention: Behavioral Risk Factor Surveillance System. National Center for Chronic Disease Prevention and Health Promotion, Division of Population Health, Atlanta, GA, 2014. http://apps.nccd.cdc.gov/brfss/index.asp.

17. Kleiner DE, Brunt EM, Van Natta M, Behling C, Contos MJ, Cummings OW, Ferrell LD, Liu YC, Torbenson MS, UnalpArida A, et al: Nonalcoholic Steatohepatitis Clinical Research Network: Design and validation of a histological scoring system for nonalcoholic fatty liver disease. Hepatology 41: 1313-1321, 2005.

18. Taylor F, Ward K, Moore TH, Burke M, Davey Smith G, Casas JP and Ebrahim S: Statins for the primary prevention of cardiovascular disease. Cochrane Database Syst Rev 19: CD004816, 2011.

19. Kiyici M, Gulten M, Gurel S, Nak SG, Dolar E, Savci G, Adim SB, Yerci O and Memik F: Ursodeoxycholic acid and atorvastatin in the treatment of nonalcoholic steatohepatitis. Can J Gastroenterol 17: 713-718, 2003. 
20. Balistreri WF: Nonalcoholic fatty liver disease - insights and controversies. CME Digestive Disease Week. Medscape, 2006.

21. Foster T, Budoff MJ, Saab S, Ahmadi N, Gordon C and Guerci AD: Atorvastatin and antioxidants for the treatment of nonalcoholic fatty liver disease: The St Francis Heart Study randomized clinical trial. Am J Gastroenterol 106: 71-77, 2011.

22. Gómez-Domínguez E, Gisbert JP, Moreno-Monteagudo JA, García-Buey L and Moreno-Otero R: A pilot study of atorvastatin treatment in dyslipemid, non-alcoholic fatty liver patients. Aliment Pharmacol Ther 23: 1643-1647, 2006.

23. Chitturi S and Farrell GC: TNF-alpha as therapeutic target in NASH: Tried, but not yet proven. J Gastroenterol Hepatol 22: 613-614, 2007.

24. Satapathy SK, Garg S, Chauhan R, Sakhuja P, Malhotra V, Sharma BC and Sarin SK: Beneficial effects of tumor necrosis factor-alpha inhibition by pentoxifylline on clinical, biochemical, and metabolic parameters of patients with nonalcoholic steatohepatitis. Am J Gastroenterol 99: 1946-1952, 2004.

25. Satapathy SK, Sakhuja P, Malhotra V, Sharma BC and Sarin SK Beneficial effects of pentoxifylline on hepatic steatosis, fibrosis and necroinflammation in patients with non-alcoholic steatohepatitis. J Gastroenterol Hepatol 22: 634-638, 2007.

26. Zein CO, Yerian LM, Gogate P, Lopez R, Kirwan JP Feldstein AE and McCullough AJ: Pentoxifylline improves nonalcoholic steatohepatitis: A randomized placebo-controlled trial. Hepatology 54: 1610-1619, 2011.
27. Van Wagner LB, Koppe SW, Brunt EM, Gottstein J, Gardikiotes K, Green RM and Rinella ME: Pentoxifylline for the treatment of non-alcoholic steatohepatitis: A randomized controlled trial. Ann Hepatol 10: 277-286, 2011

28. Adams LA,Zein CO, Angulo P and Lindor KD: A pilot trial of pen toxifylline in nonalcoholic steatohepatitis. Am J Gastroenterol 99: 2365-2368, 2004

29. Comar KM and Sterling RK: Review article: Drug therapy for non-alcoholic fatty liver disease. Aliment Pharmacol Ther 23: 207-215, 2006.

30. Neuner P, Klosner G, Schauer E, Pourmojib M, Macheiner W, Grünwald C, Knobler R, Schwarz A, Luger TA and Schwarz T: Pentoxifylline in vivo down-regulates the release of IL-1 beta, IL-6, IL-8 and tumour necrosis factor-alpha by human peripheral blood mononuclear cells. Immunology 83: 262-267, 1994.

31. Warne JP: Tumour necrosis factor alpha: A key regulator of adipose tissue mass. J Endocrinol 177: 351-355, 2003.

32. Wigg AJ, Roberts-Thomson IC, Dymock RB, McCarthy PJ, Grose RH and Cummins AG: The role of small intestinal bacterial overgrowth, intestinal permeability, endotoxaemia, and tumour necrosis factor alpha in the pathogenesis of non-alcoholic steatohepatitis. Gut 48: 206-211, 2001.

33. Ungureanu A, Gaman AE, Drocas AI, Tieranu E, Dobritoiu M The influence of steatosis and conjugate factors of response to antiviral treatment in cronic hepatitis $\mathrm{C}$, The null. Research and Science Today 11: 147-155, 2016. 